\title{
Cichorium intybus L. promotes intestinal uric acid excretion by modulating ABCG2 in experimental hyperuricemia
}

\author{
Yu Wang, Zhijian Lin, Bing Zhang ${ }^{*}$, Anzheng Nie and Meng Bian
}

\begin{abstract}
Background: Excessive production and/or reduced excretion of uric acid could lead to hyperuricemia, which could be a major cause of disability. Hyperuricemia has received increasing attention in the last few decades due to its global prevalence. Cichorium intybus L., commonly known as chicory, is a perennial herb of the asteraceae family. It was previously shown to exert potent hypouricemic effects linked with decreasing uric acid formation in the liver by down-regulating the activity of xanthine oxidase, and increasing uric acid excretion by up-regulating the renal OAT3 mRNA expression. The present study aimed to evaluate its extra-renal excretion and possible molecular mechanism underlying the transporter responsible for intestinal uric acid excretion in vivo.
\end{abstract}

Methods: Chicory was administered intragastrically to hyperuricemic rats induced by drinking $10 \%$ fructose water. The uricosuric effect was evaluated by determining the serum uric acid level as well as the intestinal uric acid excretion by HPLC. The location and expression levels of ATP-binding cassette transporter, sub-family G, member 2 (ABCG2) in jejunum and ileum were analyzed.

Results: The administration of chicory decreased the serum uric acid level significantly and increased the intestinal uric acid excretion obviously in hyperuricemic rats induced by $10 \%$ fructose drinking. Staining showed that ABCG2 was expressed in the apical membrane of the epithelium and glands of the jejunum and ileum in rats. Further examination showed that chicory enhanced the mRNA and protein expressions of ABCG2 markedly in a dose-dependent manner in jejunum and ileum.

Conclusion: These findings indicate that chicory increases uric acid excretion by intestines, which may be related to the stimulation of intestinal uric acid excretion via up-regulating the mRNA and protein expressions of ABCG2.

Keywords: Cichorium intybus L., Chicory, Hyperuricemia, Intestinal uric acid excretion, ABCG2

\section{Background}

Uric acid is the final production of purines in humans and avian species since the deficiency in uricase can lead to the elevated uric acid level in serum [1]. Hyperuricemia is characterized by high levels of sustained serum uric acid growth. It is not only one of the metabolic diseases which closely relates diabetes, hypertension, insulin resistance, obesity and other diseases, but also promotes the deposit of urate crystals in tissues arousing several pathological conditions such as acute gouty arthritis, urolithiasis and obstructive uropathy $[2,3]$. Recent studies

\footnotetext{
* Correspondence: zhangb@bucm.edu.cn

Department of Clinical Chinese Pharmacy, School of Chinese Pharmacy, Beijing University of Chinese Medicine, Beijing, China
}

show hyperuricemia has a high incidence and prevalence, and hyperuricemia is thought to be linked with the development of economy and changes of lifestyle $[4,5]$. Currently, western medicines which are used to regulating the serum uric acid level, such as allopurinol, benzbromarone, are gradually showing severe adverse reactions [6-8]. However, some clinical and experimental studies show that traditional Chinese medicine has a promising effect on lowering serum uric acid levels. A systematic review of random clinical trials from $\mathrm{Li}$ XX showed that Chinese herbal medicine may have clinical effects for functional recovery in patients with gout, and lead to a safe control of serum uric acid levels and inflammation severity [9]. Researches from Ding XQ and $\mathrm{Hu} \mathrm{QH}$ respectively showed 
that Wuling san and Simiao pill reduced the serum uric acid level significantly and enhanced urate excretion in hyperuricemic mice induced by potassium oxonate $[10,11]$. Accordingly, it must be necessary to observe the therapeutic and preventive effect of traditional Chinese medicine and explore its mechanism on hyperuricemia.

The homeostasis of uric acid levels in the body depends on the balance of its production and excretion. It is commonly accepted that two-thirds of the uric acid is excreted into urine by kidneys, and the remaining third via gut excretion $[12,13]$. As a main regulator of uric acid excretion, the kidney plays an important role in uric acid handle, including glomerular filtration, tubular reabsorption and secretion. Report indicated that the primary cause about $90 \%$ of hyperuricemia is resulted by the insufficient of renal excretion [14]. Therefore, both treatment researches and hyperuricemia mechanism studies have been widely carried out based on renal excretion. Recent research have found several important transporters involved in uric acid excretion on renal tubular, such as glucose transporter 9(Glut9, also known as SLC2A9) [15], urate transporter 1(URAT1, also known as SLC22A12) [16], organic anion transporter 1 (OAT1/NPT, also known as SLC22A6) which is the first identified member of the organic anion transporter family in the Kidney [17], and organic anion transporter 3(OAT3, also known as SLC22A8) [18]. However, intestinal excretion, another important pathway of uric acid flux, has not been studied extensively and its mechanism is still unclear. The genome-wide association studies of serum uric acid identified that ATP-binding cassette transporter, sub-family G, member 2(ABCG2, also known as BCRP) is located in a gout-susceptibility locus on chromosome 4q $[19,20]$. ABCG2 is expressed in the small intestine and the liver abundantly, and the impaired ABCG2 function is related to an increase of serum uric acid levels [18]. A recent opinion called "the Remote Sensing and Signaling Hypothesis" suggests that multi-specific SLC and ABCG2 transporters in different tissues are parts of an inter-organ and inter-organismal communication network that balances uric acid levels, metabolites and signaling molecules in the setting of an acute or chronic injury to organs [21]. The following analytical study of statistics from human supported the view that, extra-renal ABCG2 compensates to maintain uric acid homeostasis, most likely in the intestine, as renal function declines [22]. Accordingly, ABCG2 may contribute to the intestinal excretion of uric acid as extra-renal elimination pathway [23].

Cichorium intybus L., commonly known as chicory, is a perennial herb of the Asteraceae family. Chicory is well known as a cholagogic and diuretic agent in Uighur folk medicine to improve the appetite, to increase digestion and to cure liver diseases, etc. Reports showed chicory has a broad pharmacological action, including antiinflammatory, anti-oxidant, antidiabetic, anti-hyperlipidemic, hypoglycemic effects and so on [24]. In previous studies, we found chicory could lower serum uric acid effectively, and the antihyperuricemic effect of chicory mainly associates with the decreasing uric acid formation in the liver by inhibiting the activity of xanthine oxidase, and promoting uric acid excretion by up-regulating the renal OAT3 mRNA expression $[25,26]$. The above findings exhibit the potential of chicory to be developed into a therapeutic agent for treatment of hyperuricemia. However, influences of chicory on intestinal excretion of uric acid and mechanisms of chicory-mediated hyporuricemic action have not been completely clarified. Therefore, the present investigation was aimed at evaluating the uricosuric action via intestinal excretion pathway. The potential molecular mechanism of its excretion action was investigated by determining protein and mRNA expression levels of ABCG2 in the small intestine in hyperuricemic rats treated with chicory.

\section{Methods \\ Drugs}

Chicory used in the study was authenticated by Professor Yong-Hong Yan (Traditional Chinese Medicine Appraisal Teaching and Research Section of Beijing University of Chinese Medicine). Chicory was grinded into powder and weighed. The powder was extracted with water $(1 \mathrm{~g}: 10 \mathrm{ml})$ by heating to reflux for $1 \mathrm{~h}$ twice. Then the solution was concentrated by a rotary evaporator after filtering, and diluted to different volume with purified water [27]. Benzbromarone tablets were obtained from Heumann Pharma GmbH (Germany).

\section{Experimental animals}

Ninety-six male Sprague-Dawley rats of SPF grade, weighting 230-250 g were obtained from Beijing SPF Laboratory Animal Technology co., LTD (Certificate of Quality: SCXK-2011-0004). The animals were maintained in plastic cages with water and food available ad libitum. All animals were housed on a $12 \mathrm{~h}$ day-night cycle and the temperature and humidity were kept at $22-24{ }^{\circ} \mathrm{C}$ and $50 \%$, respectively. All efforts were made to minimize animal suffering and to keep the number of animals used to a minimum. The study protocol was approved by the Animal Care and Ethics Committee in Beijing University of Chinese Medicine.

\section{Induction of hyperuricemia and drug treatment}

Hyperuricemic rats were generated by giving $10 \% \mathrm{D}$ fructose (AMRESCO, USA) in drinking water as previously described [28]. The rats were divided randomly into six groups of 16 rats each, which were CON (the control group was given normal drinking water), MOD 
(the model group was given 10\% fructose in drinking water), BEN (the benzbromarone-treated group was given $10 \%$ fructose in drinking water and treated with $20 \mathrm{mg} \cdot \mathrm{kg}-1 \cdot \mathrm{d}-1$ benzbromarone water solution by intragastric administration, using a ball-tip needle), $\mathrm{CHI}-\mathrm{H}$ (the high-dosage chicory treated group was given 10\% fructose in drinking water and treated with $16.7 \mathrm{~g} \cdot \mathrm{kg}$ $1 \cdot d-1$ chicory water solution by intragastric administration, using a ball-tip needle), CHI-M (the middle-dosage chicory treated group was given $10 \%$ fructose in drinking water and treated with $13.3 \mathrm{~g} \cdot \mathrm{kg}-1 \cdot \mathrm{d}-1$ chicory water solution by intragastric administration, using a ball-tip needle), CHI-L (the low-dosage chicory treated group was given $10 \%$ fructose in drinking water and treated with $6.6 \mathrm{~g} \cdot \mathrm{kg}-1 \cdot \mathrm{d}-1$ chicory water solution by intragastric administration, using a ball-tip needle). During the experiment, the CON group and the MOD group were intragastricly treated with saline.

\section{Effects of chicory on serum uric acid levels}

The hyperuricemic rats were induced by the above method. All drugs were given Intragastric administration once a day. Body weight of rats was recorded every 10 days. Blood samples were taken from rats by cutting the trail tips after a $12 \mathrm{~h}$ fasting. Then centrifuge the blood samples at $3500 \mathrm{rpm}$ for $10 \mathrm{~min}$, at $4{ }^{\circ} \mathrm{C}$ for plasma separation for later analysis. After a forty-day treatment of the experiment, half of animals in each group were killed and then dissected after $12 \mathrm{~h}$ fasting, while the other part of rats in each group were continued to treat with drugs and carried on the intestinal uric acid excretion experiment in a period of 10 days in parallel. Serum uric acid levels were detected according to the uric acid assay kit (BioSino Bio-Technology \& Science Inc., China).

\section{Effects of chicory on intestinal uric acid excretion by HPLC}

The analysis of Intestinal uric acid excretion was carried out by the modified method previously described and made an update [29]. Rats were anaesthetized by intraperitoneal injection of pentobarbital sodium and cannulated with polyethylene tubing at the upper duodenum and the middle jejunum to make an intestinal loop at the upper half of the intestine after the overnight fasting. The intestinal contents were removed by injecting saline and air slowly. Then the efflux buffer (saline containing $0.3 \mathrm{mM}$ potassium oxonate) was introduced into the intestinal loop using peristaltic pump at the speed of intestinal tract movement for $2 \mathrm{~h}$. After the set time, the efflux buffer in the loop was collected and the length of the whole small intestine and the intestine loop were measured. The concentration of uric acid was quantified by HPLC. Intestinal uric acid excretion was calculated by the following equation: [Intestinal uric acid excretion] $=$ [Uric acid concentration in the intestinal loop] $\times$ [Volume of efflux buffer in the intestine loop] $\times$ [Length of the whole small intestine]/[Length of the intestinal loop].

Uric acid levels in the efflux buffer were analyzed by HPLC method by using a reverse phase $\mathrm{C} 18$ column $(4.6 \mathrm{~mm} \times 250 \mathrm{~mm}, 5 \mu \mathrm{m}$,Agilent $)$ on a Shimadzu HPLC system, operated at $30{ }^{\circ} \mathrm{C}$. The mobile phase was composed of $0.2 \%$ acetic acid water solution and $\mathrm{MeOH}$ system. The samples were filtered by $0.22 \mu \mathrm{m}$ microfiltration membrane after suitable dilution. Twenty microliter of the treated sample was injected into the column and eluted with the mobile phase at the flow rate of $1 \mathrm{~mL} / \mathrm{min}$. The eluate was monitored for absorbance at $288 \mathrm{~nm}$. External standard method of uric acid standard (purity $\geq 99 \%$, Sigma-aldrich, USA) was used in this study.

\section{Measurement effects of chicory on mRNA expressions of $A B C G 2$ in rats by quantitative real-time polymerase chain reaction ( $\mathrm{qPCR}$ )}

The tissues of jejunum and ileum $(100 \mathrm{mg})$ in rats were homogenized in $1 \mathrm{~mL}$ Trizol reagent (Thermo Scientific, USA) to extract total RNA. RNA integrity was evaluated by electrophoresis in 1\% agarose gel, and the concentration of total RNA was measured with a trace nucleic acid quantitative instrument. The extracted RNA was reverse-transcribed following the manufacturer s protocol of RevertAid First Strand cDNA Synthesis Kit (Thermo Scientific, USA). Quantitative real-time PCR was performed using Universal SYBR Green Supermix (Bio-rad) and CFX96 (BIO-RAD) at $95{ }^{\circ} \mathrm{C}$ 30s followed by 40 cycles at $95{ }^{\circ} \mathrm{C}$ for $5 \mathrm{~s}$, and $60{ }^{\circ} \mathrm{C}$ for $30 \mathrm{~s}$. The sense and antisense primers for ABCG2 were designed according to the mRNA sequence (GenBank accession number NM_181381). Amplification of PCR fragments spanning different exons was used to prevent contamination by genomic DNA. The sense primer used was 5'- GGC CTG GAC AAA GTA GCA GA-3', and the antisense primer was 5'- GTT GTG GGC TCA TCC AGG AA-3'. The sense and antisense primers for GAPDH were according to literature report [24]. The sense primer used was 5'- GGT GGA CCT CAT GGC CTA CA-3', and the antisense primer was 5' - ATT GTG AGG GAG ATC CTC AGT GT-3'. The ABCG2 mRNA expression values were normalized to GAPDH expression.

\section{Location of ABCG2 and effects of chicory on ABCG2 protein expressions in rats by immunohistochemistry}

Formaldehyde-fixed paraffin-embedded tissues of jejunum and ileum were deparaffinized in xylene and rehydrated. Paraffin sections were pretreated with $10 \mathrm{mM}$ sodium citrate buffer solution ( $\mathrm{pH}$ 6.0), then cool them to room temperature to unmask antigen. After rinsing by PBS for three times, endogenous peroxidase activity was blocked 
in dark using 3\% hydrogen peroxide for $12.5 \mathrm{~min}$. Before staining, the slides were first incubated with 5\% normal goat serum for $25 \mathrm{~min}$. All of above operations were at room temperature. Slides should be washed before the next step. Subsequently, paraffin sections were incubated with a 1:200 dilution of ABCG2 polyclonal antibody (B-25, Santa Cruz, USA) at $4{ }^{\circ} \mathrm{C}$ overnight. B-25 was diluted in the antibody diluent buffer.

In this case, after incubation with ABCG2 polyclonal antibody and placed them in the incubator for $60 \mathrm{~min}$ to return to $37{ }^{\circ} \mathrm{C}$, slides were incubated for $60 \mathrm{~min}$ with polymer auxiliary agent, subsequently incubated for $30 \mathrm{~min}$ with HRP-conjugated goat anti-rabbit IgG (ZSGB Biotechnology, China). All above behaviors were acted at $37{ }^{\circ} \mathrm{C}$, and slides should be rinsed with PBS thrice for $5 \mathrm{~min}$ before the next step. Color development was achieved with a solution-containing DAB kit (ZSGB Biotechnology, China), incubated for $3 \mathrm{~min}$, and rinsed under running water. After counterstaining with hematoxylin, slides were mounted. For each type of tissue, negative control were included, i.e., by omission of the primary antibody by using with PBS.

All measurements were performed with the automated upright microscope system (Olmpus BX53), and five random images from each section obtained from six rats were randomly captured by high-speed color CCD camera (Olmpus DP72CCD). Imagine Pro-Plus6.0 software was used to analyze pictures. The positive immunostained area in a total area under an image field of each section was calculated.

\section{Measurement effects of chicory on protein expressions of ABCG2 in rats by western blotting method}

After weighing, jejunum and ileum samples from each group were homogenized with ball-grinding mill in radioimmunoprecipitation assay lysis buffer containing phenylmethanesulfonyl fluoride (Solarbio Life Sciences, China) and bathed on ice for $5 \mathrm{~min}$. Then the lysate was centrifuged at $12000 \mathrm{rpm}, 4{ }^{\circ} \mathrm{C}$ for $10 \mathrm{~min}$ to extract total proteins. BCA method (Solarbio Life Sciences, China) was performed to determining the amount of the proteins. The total proteins were diluted at suitable multiple and incubated with $4 \times$ sodium dodecyl sulfate (SDS) loading buffers in the boiling water for $10 \mathrm{~min}$.
For western blot analysis, $60 \mu \mathrm{g}$ of treated sample was separated on a $10 \%$ SDS-polyacrylamide gel electrophoresis plate with a $5 \%$ stacking gel. Proteins were transferred electrophoretically onto polyvinylidene difluoride (PVDF) membrane (Millipore, Germany) using a blotter (LIUYI Laboratories) at $300 \mathrm{~mA}$ for $1.5 \mathrm{~h}$. The membrane was blocked with Tris-buffered saline containing $0.1 \%$ Tween20 (TBST) and 5\% skimmed milk powder for $2 \mathrm{~h}$ at room temperature. The membrane was incubated with rabbitanti-ABCG2 antibody (1:400, B-25, Santa Cruz) and mouse-anti- $\beta$-actin antibody $(1: 15,000,60,008-1$, Proteintech, USA) in TBST containing 5\% skimmed milk powder overnight at $4{ }^{\circ} \mathrm{C}$. After washing the membranes three times with TBST, immunoreactive bands were detected using HRP conjugated Goat Anti-Rabbit IgG (1:10,000, ZB-2301, ZSGB Biotechnology, China) or Goat AntiMouse IgG (1:15,000, SA00001-1, Proteintech, USA) as the secondary antibody in TBST for $1.5 \mathrm{~h}$ at room temperature. The proteins were visualized using enhanced chemiluminescence (ECL) reagent (Millipore, Germany). The density of bands was analyzed by Image J and normalized to $\beta$-actin.

\section{Statistical analysis}

The data were expressed as the mean \pm S.E. The statistical analysis was performed using an one-way ANOVA followed by the Dunnett's multiple comparison tests to determine levels of significance by SPSS20.0 software. A $P$-value of $P<0.05$ was considered statistically significant.

\section{Results}

\section{Growth performance}

Rats were in good status and the body weight was increased steadily throughout the entire experimental period. As showed in Table 1, there was no significant difference in the body weight among the six groups during the experimental period.

\section{Effects of chicory on serum uric acid levels}

The $10 \%$ fructose-drinking caused a significant increase in serum uric acid levels, and this enhancement could be maintained for 40d steadily (Table 2). As showed in Table 2, chicory given intragastrically at the high and the middle doses once a day for $10 \mathrm{~d}$ could attenuated serum

Table 1 Body weight of rats during experimental days $(n=16, g)$

\begin{tabular}{llllll}
\hline Groups & Od & 10d & 20d & 30d & 40d \\
\hline CON & $251.31 \pm 10.79$ & $324.79 \pm 14.79$ & $358.12 \pm 14.11$ & $398.38 \pm 21.24$ & $422.49 \pm 25.14$ \\
MOD & $248.38 \pm 11.02$ & $311.45 \pm 19.66$ & $347.19 \pm 22.97$ & $387.68 \pm 27.10$ & $416.23 \pm 32.75$ \\
BEN & $243.45 \pm 10.01$ & $312.36 \pm 11.58$ & $344.04 \pm 14.44$ & $383.26 \pm 18.52$ & $405.41 \pm 19.26$ \\
CHI-H & $248.57 \pm 11.19$ & $316.72 \pm 23.56$ & $357.91 \pm 27.60$ & $397.21 \pm 33.74$ & $418.50 \pm 40.09$ \\
CHI-M & $247.94 \pm 14.50$ & $308.68 \pm 22.28$ & $347.85 \pm 26.18$ & $391.55 \pm 32.40$ & $412.93 \pm 37.68$ \\
CHI-L & $246.23 \pm 12.16$ & $315.35 \pm 19.69$ & $356.63 \pm 22.61$ & $395.39 \pm 25.87$ & $417.62 \pm 30.46$ \\
\hline
\end{tabular}


Table 2 Uric acid-lowering effects of intragastric chicory in the hyperuricemic rats $(n=16, \mu \mathrm{mol} / \mathrm{L})$

\begin{tabular}{llllll}
\hline Groups & Od & $10 d$ & $20 d$ & $30 d$ & $40 d$ \\
\hline CON & $51.47 \pm 21.30$ & $66.44 \pm 26.12$ & $76.22 \pm 22.57$ & $133.80 \pm 33.23$ & $74.10 \pm 24.41$ \\
MOD & $56.72 \pm 28.76$ & $87.63 \pm 27.34^{*}$ & $98.07 \pm 22.23^{* *}$ & $177.08 \pm 44.99^{* *}$ & $95.80 \pm 18.01^{* *}$ \\
BEN & $50.56 \pm 26.81$ & $74.66 \pm 35.29$ & $75.64 \pm 14.69^{\# \#}$ & $147.11 \pm 30.84^{\#}$ & $82.60 \pm 19.76$ \\
CHI-H & $50.33 \pm 21.91$ & $52.40 \pm 16.77^{\# \#}$ & $66.17 \pm 21.09^{\# \#}$ & $145.61 \pm 36.58^{\#}$ & $73.82 \pm 35.90^{\#}$ \\
CHI-M & $53.08 \pm 25.51$ & $55.90 \pm 29.62^{\# \#}$ & $68.82 \pm 16.84^{\# \#}$ & $112.52 \pm 45.48^{\# \#}$ & $83.07 \pm 41.07$ \\
CHI-L & $50.22 \pm 21.67$ & $70.05 \pm 32.18$ & $65.09 \pm 28.36^{\# \#}$ & $155.27 \pm 44.47$ & $98.11 \pm 9.46$ \\
\hline
\end{tabular}

Note: ${ }^{*} P<0.05,{ }^{* * *} P<0.01$ vs. the CON group; ${ }^{\#} P<0.05$, ${ }^{\# \#} P<0.01$ vs. the MOD group. Means with different superscript lowercase letters in the same column are significantly different

uric acid levels significantly, moreover the reduced serum uric acid levels could be kept effectively during the whole experiment in fructose-induced hyperuricemic rats when compared to the untreated hyperuricemic rats $(P<0.05)$. The low dose of chicory reduced serum uric acid levels markedly after administrating for $20 \mathrm{~d}$ $(P<0.05)$, but did not show significant differences during the subsequent experimental days when compared with the untreated hyperuricemic rats. The onset of lowering serum uric acid levels in the benzbromarone group was from the 20th day to the 30th day $(P<0.01$, $P<0.05)$ when compared with the untreated model group in this experiment.

\section{Effects of chicory on intestinal uric acid excretion by HPLC}

As shown in Table 2 and Fig. 1, 10\% fructose-drinking increased uric acid levels in serum significantly as well as decreased the excretion of intestinal uric acid obviously in rats, when compared with the normal control group $(P<0.05)$. Administration of chicory reduced serum uric acid levels significantly in a dose-dependent manner to some degree and enhanced the intestinal uric acid excretion at the high $\operatorname{dose}(P<0.01)$, middle dose and low $\operatorname{dose}(P<0.05)$ compared with the model group exhibiting the promotion of uric acid excretion in gut. As a positive control, benzbromarone showed a remarkable reduction of serum uric acid but there was no significant change of uric acid excretion of the intestine compared with hyperuricemic rats.

\section{Effects of chicory on ABCG2 mRNA expressions in jejunum and ileum}

Effects of chicory and benzbromarone on ABCG2 mRNA expressions of jejunum and ileum are shown in Fig. 2, from which it can be seen that fructose-induced hyperuricemic rats showed the remarkable downregulation of ABCG2 mRNA expressions in jejunum and ileum $(P<0.05, P<0.05)$, when compared with the normal controlled rats. Chicory up-regulated the expression of ABCG2 mRNA was shown in a dose-dependent manner. The high dose of chicory significantly increased ABCG2 mRNA expressions in jejunum $(P<0.05)$ as well as ileum $(P<0.05)$, when compared with the model

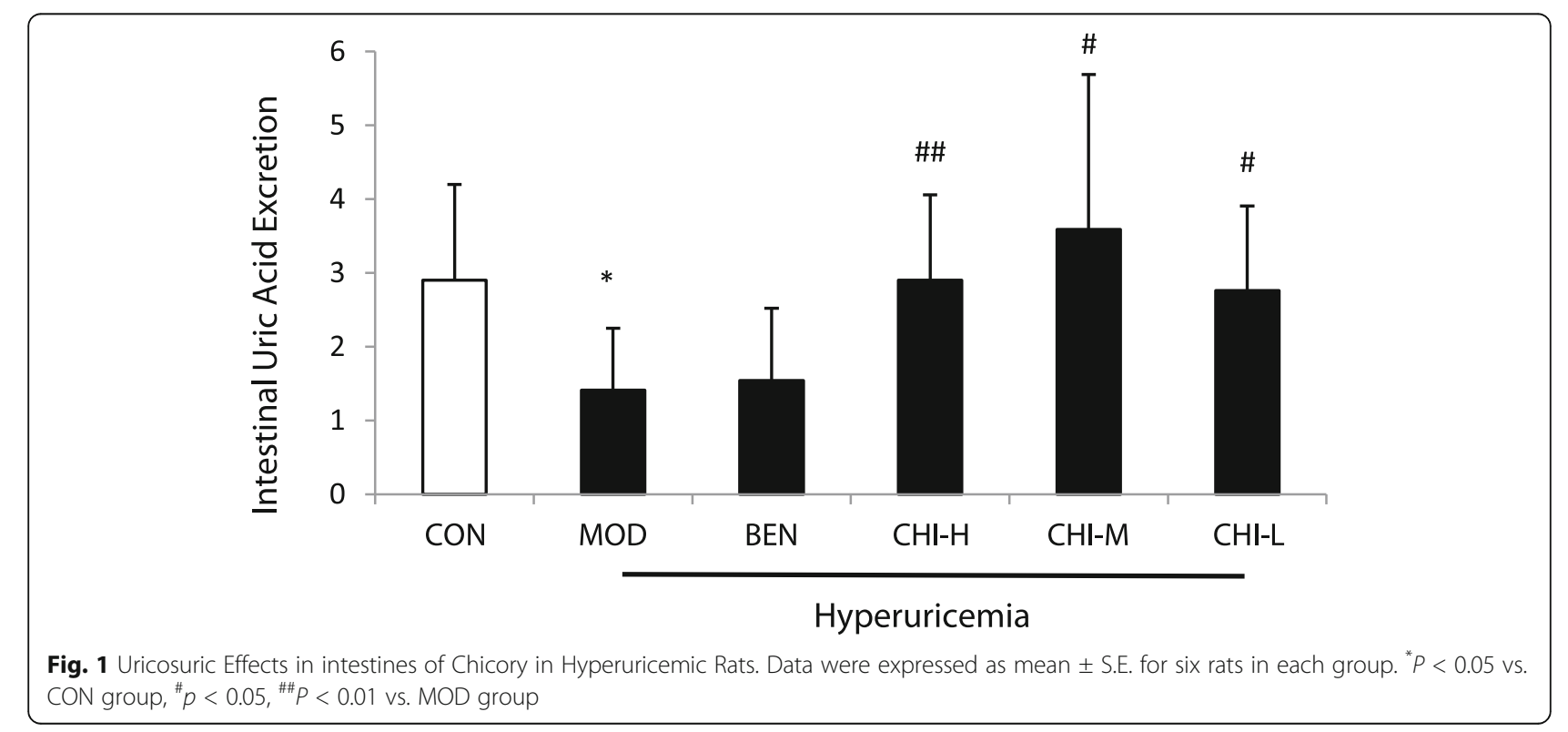



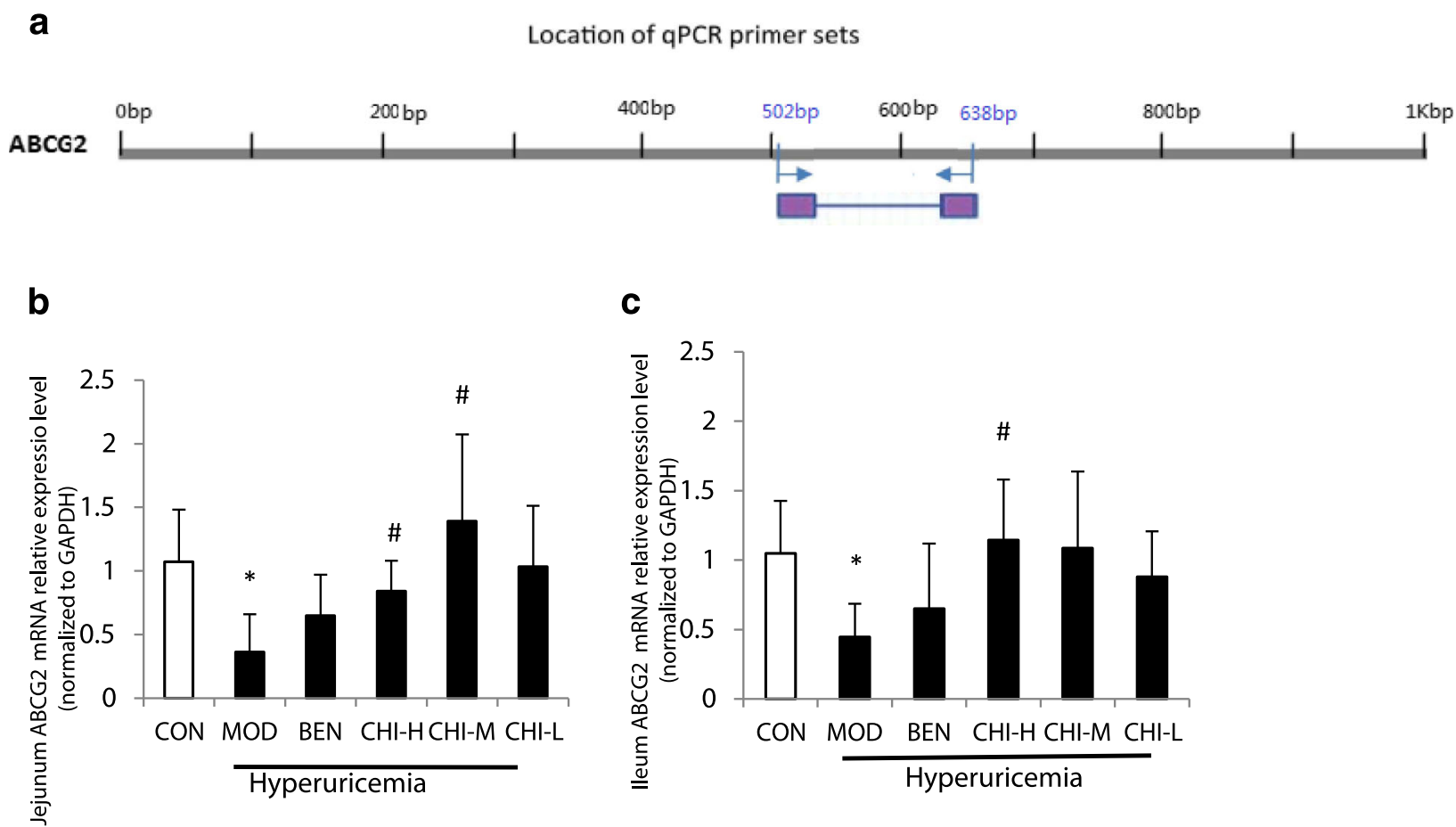

Fig. 2 Effects of Chicory on ABCG2 mRNA Expressions. a lllustration of the location of primer sets for qPCR amplification of ABCG2 mRNA transcripts. Numbers refer to the translation start site of ABCG2. b In jejunum. $\mathbf{c}$ In ileum. Data were expressed as mean \pm S.E. for six rats in each group. ${ }^{*} P<0.05$ vs. CON group, ${ }^{\#} p<0.05$ vs. MOD group

group. The middle dose of chicory markedly up-regulated ABCG2 mRNA expressions in jejunum $(P<0.05)$, but had no significant change in ileum, when compared with the model group. There were no remarkable difference in the expression of ABCG2 mRNA between the low dose of chicory group and the model group in jejunum or ileum. Similarly, there was no change of ABCG2 mRNA expressions in jejunum still ileum was observed in between benzbromarone and hyperuricemic rats.

\section{Location of ABCG2 and effects of chicory on ABCG2 protein expressions in jejunum and ileum in rats by immunohistochemistry}

There was no immunoreactivity in the sections set aside for negative control and ABCG2 positivity was indicated by cytoplasmic golden brown staining. As shown in Fig. 3, prominent staining was observed in the small intestine, with strong apical staining of the epithelium and glands of the jejunum and ileum. From the Table 3, ABCG2 protein expressions of the model group were reduced significantly in the jejunum and ileum $(P<0.05, P<0.01)$, when compared to the control group. An evaluation of ABCG2 protein expressions in all chicory-treated groups was notably higher in the jejunum and ileum compared with the model group $(P<0.01)$, whereas the benzbromarone-treated group showed pronounced increase only in the ileum $(P<0.01)$.
Effects of chicory on ABCG2 protein expressions in jejunum and ileum in rats by western blotting

Effects of chicory and benzbromarone on protein expressions of ABCG2 in hyperuricemic rats are shown in Figs. 4 and 5, from which it can be seen that fructoseinduced hyperuricemic rats showed the remarkable reduction of ABCG2 protein expressions in jejunum and ileum $(P<0.05, P<0.05)$, when compared with the normal control rats. It also showed that the protein expression levels of each treatment group were elevated in varying degrees in jejunum and ileum by comparing with the hyperuricemic rats. Chicory could increase ABCG2 protein expression, as well as in the ileum $(P<0.01$, $P<0.01)$, compared with the model group. However, there was no obvious alteration between the low dose of chicory group and the model group in jejunum and ileum. Similarly, there was no change of the ABCG2 protein expression in jejunum still ileum was observed between benzbromarone and hyperuricemic rats.

\section{Discussion}

Pathomechanism of hyperuricemia induced by fructose Fructose is widely used in the food industry for its flavor [30]. However, with the growth of fructose consumption, we also witnessed its negative influences. There are more and more speculations that excessive consumption of fructose could cause insulin resistance, obesity, and 
a

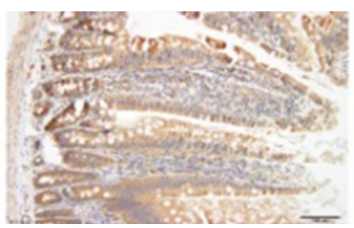

d

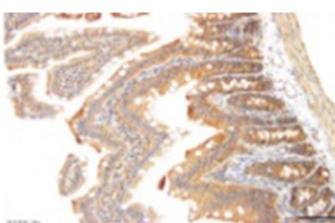

g

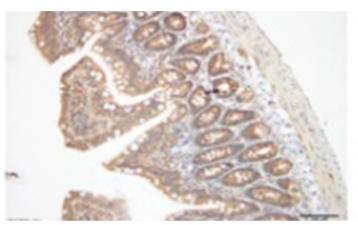

j

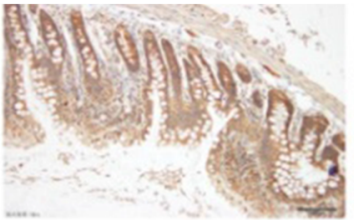

b

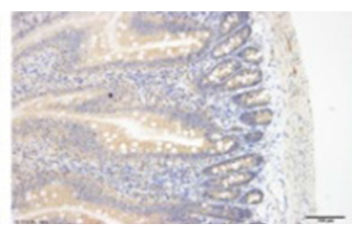

e

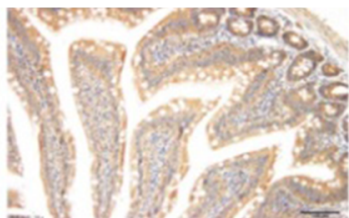

h

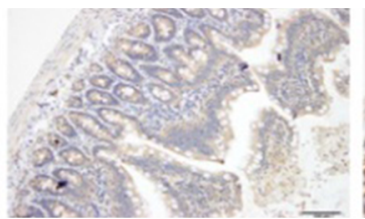

k

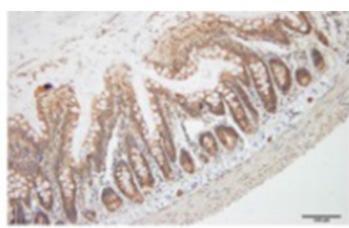

C

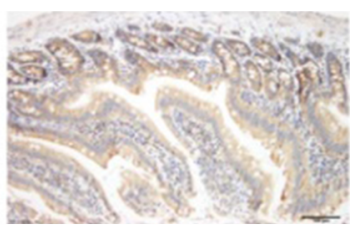

f

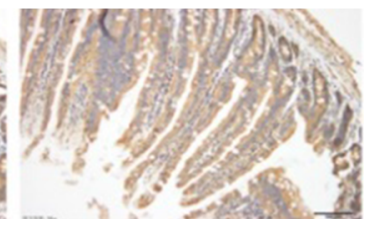

i

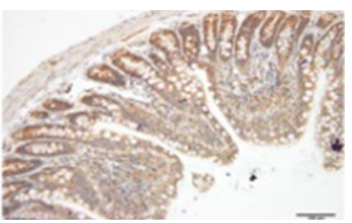

I

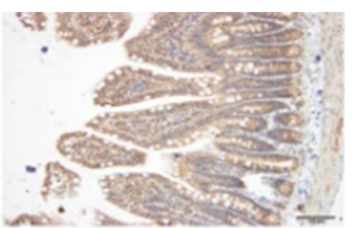

Fig. 3 Location of ABCG2 and effects of chicory on ABCG2 protein expression. a Jejunum of normal rat ABCG2 IHC stain ( $\times 20$ objective lens). $\mathbf{b}$ Jejunum of model rat ABCG2 IHC stain ( $\times 20$ objective lens). c Jejunum of benzbromarone rat ABCG2 IHC stain ( $\times 20$ objective lens). $\mathbf{d}$ Jejunum of chicory extract high-dose rat ABCG2 IHC stain ( $\times 20$ objective lens). e Jejunum of chicory extract middle-dose rat ABCG2 IHC stain ( $\times 20$ objective lens). $\mathbf{f}$ Jejunum of chicory extract low-dose rat ABCG2 IHC stain ( $\times 20$ objective lens). $\mathbf{g}$ lleum of normal rat ABCG2 IHC stain ( $\times 20$ objective lens). $\mathbf{h}$ lleum of model rat ABCG2 IHC stain ( $\times 20$ objective lens). $\mathbf{i}$ lleum of benzbromarone rat ABCG2 IHC stain ( $\times 20$ objective lens). $\mathbf{j}$ lleum of chicory extract high-dose rat ABCG2 IHC stain ( $\times 20$ objective lens). $\mathbf{k}$ lleum of chicory extract middle-dose rat ABCG2 IHC stain ( $\times 20$ objective lens). I lleum of chicory extract low-dose rat ABCG2 IHC stain ( $\times 20$ objective lens). Low expression of model group in jejunum and ileum $(P<0.05$, $P<0.01$ vs. control group slices), which developed heavy stains; inhibition of ABCG2 by chicory $(P<0.01, P<0.01$ vs. model group slices)

Table 3 Effects of Chicory on the ABCG2 protein Expressions in jejunum and ileum by immunohistochemistry $(n=6)$

\begin{tabular}{|c|c|c|c|c|}
\hline \multirow[b]{2}{*}{ Groups } & \multicolumn{2}{|l|}{ Jejunum } & \multicolumn{2}{|l|}{ Ileum } \\
\hline & Positive area $\left(10^{5}\right)$ & Accumulated integrated optical density $\left(10^{4}\right)$ & Positive area $\left(10^{5}\right)$ & Accumulated integrated optical density $\left(10^{4}\right)$ \\
\hline $\mathrm{CON}$ & $4.68 \pm 2.11$ & $2.16 \pm 1.44$ & $12.56 \pm 0.66$ & $4.24 \pm 0.49$ \\
\hline MOD & $3.41 \pm 1.77^{*}$ & $1.31 \pm 0.81^{*}$ & $12.90 \pm 0.83$ & $3.72 \pm 0.77^{* *}$ \\
\hline BEN & $4.17 \pm 1.70$ & $1.82 \pm 1.12$ & $12.82 \pm 0.60$ & $4.49 \pm 0.65^{\# \#}$ \\
\hline $\mathrm{CHI}-\mathrm{H}$ & $4.77 \pm 1.27^{\# \#}$ & $2.00 \pm 0.48^{\# \#}$ & $13.82 \pm 0.29^{* * \# \#}$ & $8,87 \pm 2.03^{* * \# \#}$ \\
\hline $\mathrm{CHI}-\mathrm{M}$ & $5.27 \pm 1.02^{\# \#}$ & $2.12 \pm 0.49^{\# \#}$ & $13.82 \pm 0.42^{* * \# \#}$ & $7.46 \pm 0.51^{* * \# \#}$ \\
\hline CHI-L & $4.26 \pm 1.40^{\#}$ & $1.87 \pm 0.84^{\#}$ & $13.88 \pm 0.18^{* * \# \#}$ & $7.54 \pm 0.62^{* * \# \#}$ \\
\hline
\end{tabular}

Note: ${ }^{*} P<0.05,{ }^{* *} P<0.01$ vs. CON group; ${ }^{\#} P<0.05,{ }^{\# \#} P<0.01$ vs. MOD group. Means with different superscript lowercase letters in the same column are significantly different 


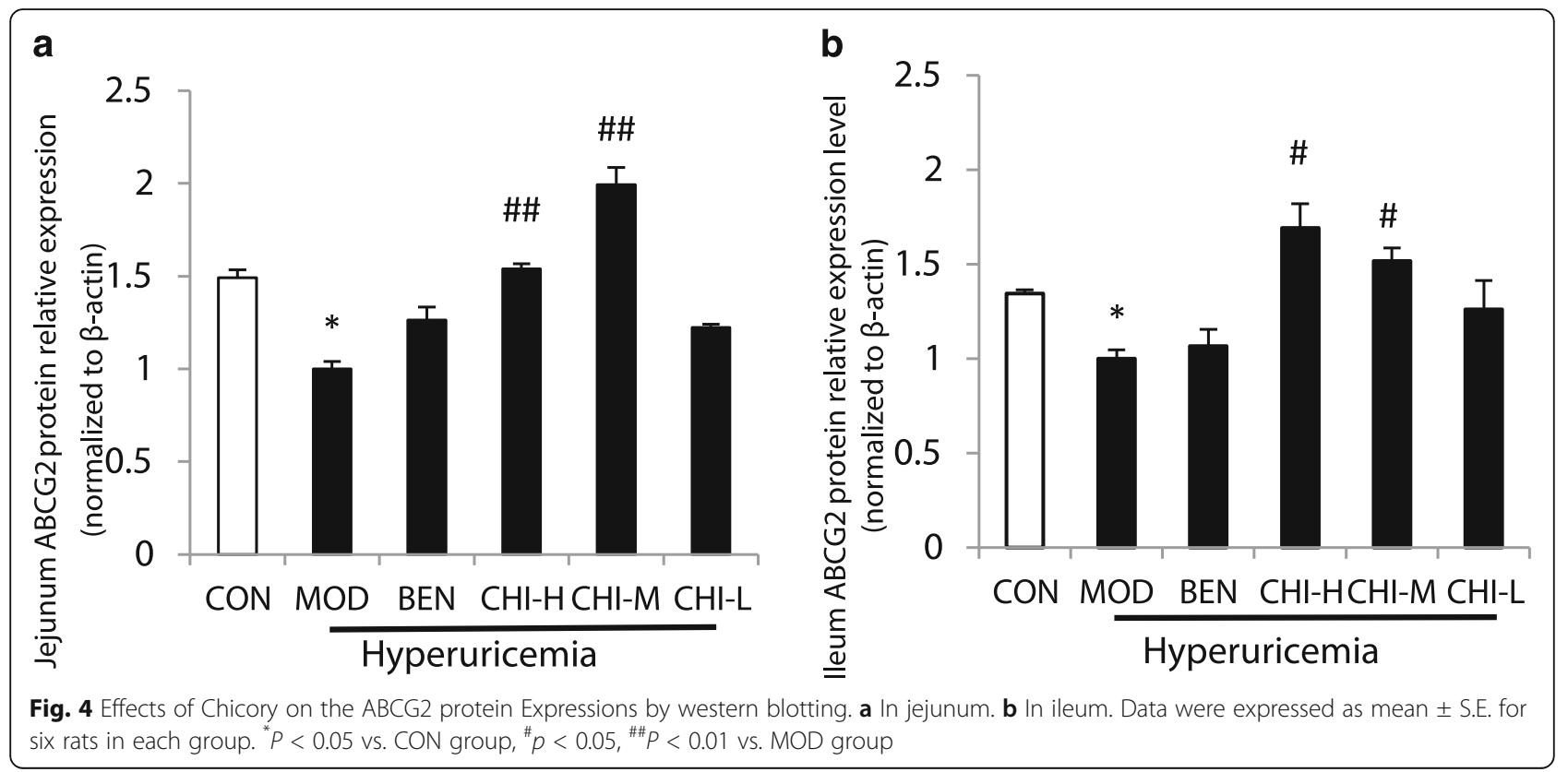

have a strong correlation with hyperuricemia [31, 32]. A rapid and sustained enhancement of serum uric acid levels in humans will happen after the oral or intravenous ingestion of fructose, especially in gout patients [33, 34]. Recent animal experiments proved that sustained or excessive intake of fructose could cause the abnormal serum uric acid levels [35]. As expected, our studies showed that the drinking of fructose with the concentration of $10 \%$ for 10d induced the elevation of serum uric acid levels markedly and maintained steadily in rats during the whole experiment (Table 2). The mechanism of induction of hyperuricemia by fructose is controversial. It is commonly accepted that the cause of hyperuricemia induced by fructose is associated with the formation of urate [36, 37]. In addition, Abdulla, M.H., et al., found that the high dose of fructose intake altered renal haemodynamic and excretory function [38]. Our former study mentioned previously also showed the high dose of fructose had an effect on renal dysfunction. Nevertheless, the change of intestinal uric acid excretion in fructose-induced hyperuricemia is still unclear.

\section{The role of the intestine in hyperuricemia}

Hyperuricemia is traditionally classified into the 'overproduction' type, the 'underexcretion' type, and the 'combined' type. This classification is solely based on the amount of liver uric acid formation and renal urate excretion, and the extra-renal excretion such as gut excretion is not taken into consideration. The process of uric acid handling in the liver and kidneys has been carried out widely, while the mechanism of uric acid elimination in the intestine is poorly understood. Matsuo, $\mathrm{H}$., et al. suggested the 'overproduction' type in the current concept of hyperuricemia could be renamed 'renal overload'

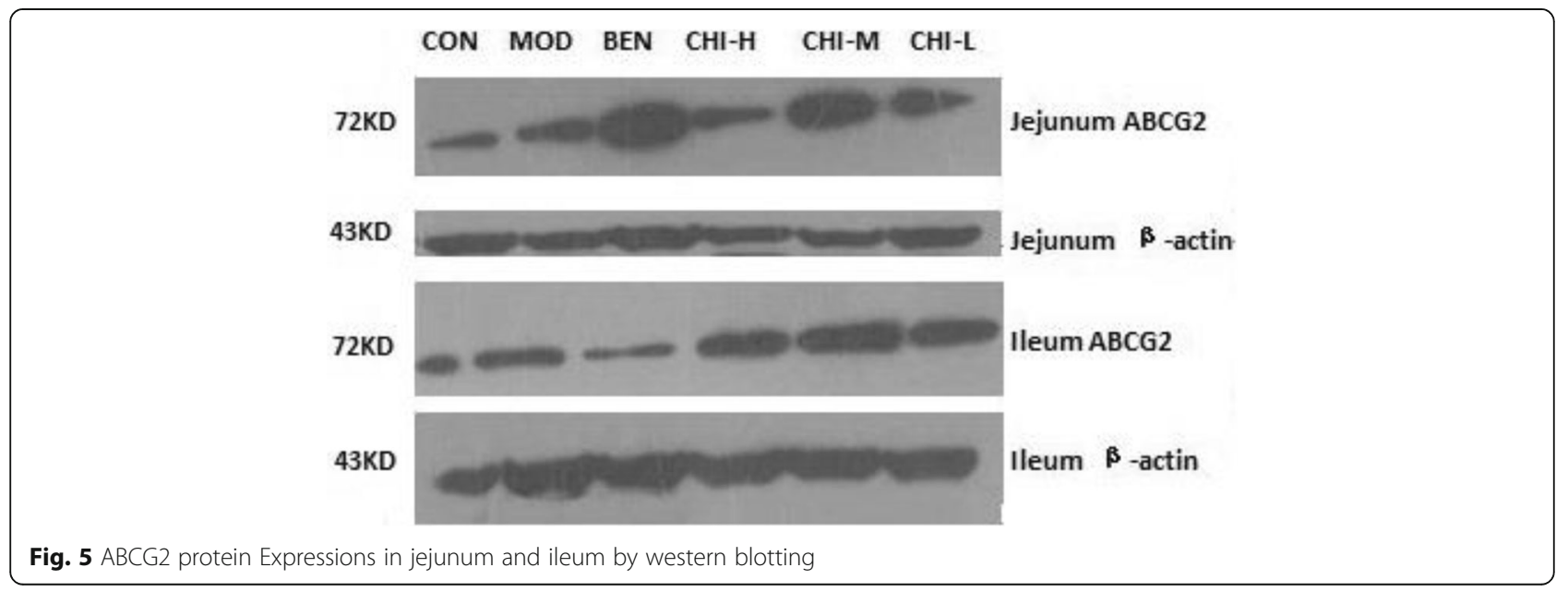


type, which consists of 'extra-renal urate underexcretion' and genuine 'urate overproduction' type [29]. Their research provided a new concept which is valuable for the understanding of hyperuricemia and emphasized the decreased intestinal urate excretion is a common cause of hyperuricemia. In our study, the intestinal uric acid excretion reduced significantly in hyperuricemia rats when compared with the normal group (Fig. 1). This illustrated that the mechanism of hyperuricemia induced by fructose would be associated with excretion of uric acid in the intestine.

\section{The medical function of chicory on hyperuricemia}

Chicory is widely used as a leafy vegetable, forage grass, raw materials of sugar and coffee substitute materials and it has been a Uighur and Mongolian folk medicine for many years. Its main pharmacological effects include hypoglycemic action, antihyperlipidemic action, hypouricemic action, hepatoprotective effect and the adjustive effect of the digestive system and cardiovascular system [39]. All above indicate that chicory is potential and exploitable in healthy beverages, functional foods and miracle drugs. In the previous study, chicory could significantly decrease serum uric acid levels by inhibiting the activities of uricopoiesis metabolic enzymes of $5^{\prime}$-nucleotidase, adenosine deaminase, purine nucleoside phosphorylase, guanine deaminase and xanthine oxidase. This is an indication that chicory could decrease the formation of uric acid in the liver [40]. In addition, our research also displayed that chicory could lower the serum uric acid levels obviously by upregulating the renal OAT3 mRNA expression, exhibiting that the hyporicemic effects of chicory could be associated with promoting excretion of uric acid in the kidney [25]. These findings suggested that the hypouricemic action of chicory might be related not only to the inhibition of uric acid formation in the liver, but also the enhancement of renal uric acid excretion. The potent hypouricemic action of chicory in vivo could be explained by the dual actions of production and excretion. In the current study, the high and middle doses of chicory were reduced serum uric acid levels significantly in fructose-induced hyperuricemia rats (Table 2). The uricosuric action of chicory was earlier and more stable than benzbromarone. To further determine if the uricosuric effect observed is linked with the excretion of uric acid in the intestine, we investigated the effect of chicory on the intestinal uric acid excretion in hyperuricemia rats induced by fructose consumption. Administration of chicory once a day for $10 \mathrm{~d}$ could significantly decrease serum uric acid levels and increase the intestinal uric acid excretion at the high and middle dosages in hyperuricemia rats (Fig. 1). Those results illustrated that chicory has the ability of accelerating intestinal uric acid excretion and is a potent uricosuric agent.

\section{The role of $A B C G 2$ in hyperuricemia and the regulatory effect by chicory}

ABCG2 is a member of the ATP-binding cassette transporter superfamily and has only one ATP-binding cassette and six putative transmembrane domains. This suggests that ABCG2 is a half-transporter, which may function as a homodimer or heterodimer. Recent research showed that the elevated serum uric acid level is associated with decreased ABCG2 activity which is resulted by the genetic polymorphisms [41]. A genome-wide association study (GWAS) of serum uric acid levels proved that ABCG2 has multiple SNPs in a genomic region on chromosome 4 as associated with urate levels and prevalence of gout [42]. ABCG2 is expressed on the apical membrane in several tissues, including liver, intestines and kidneys [43], but the expression of ABCG2 is much higher in the intestinal epithelial cells and hepatocytes than in proximal tubular cell [44]. It has been shown to transport a wide range of substrates, such as nucleoside analogue drug lamivudine, which are structurally similar to urate, fluorochrome rhodamine, folic acid etc. [45]. There is study shown that the ABCG2 is a high-capacity urate secretion transporter [41]. Kolz, M., et al., conducted a meta-analysis of genome-wide association scans from 14 studies totaling 28,141 participants of European descent and the result indicated ABCG2 is closely related to the concentration of serum uric acid [46]. Hosomi A., et al., found that the expression of rABCG2 mRNA was well correlated with uric acid secretory activity into the intestinal lumen [23]. Yano, $\mathrm{H}$., et al., found the urine UA excretion and UA clearance were decreased significantly in a 5/6 nephrectomy rat, whereas the serum uric acid level was not significant, and the expression of ABCG2 in the ileum of the nephrectomy group showed significant upregulation [47]. This suggests that an excretory pathway other than the kidney, probably the intestine, may operate in a complementary role. From the above, ABCG2 would be critical to accelerating intestinal uric acid excretion and has potential to lower serum uric acid.

In the current study, significant staining was observed in jejunum and ileum with strong apical staining of the epithelium as well as gland (Fig. 3). From the analysis of immunohistochemical figures (Table 3), we observed that ABCG2 protein expressions in jejunum and ileum were prominently decreased in the rat model obtained the fructose drinking. The significant promoted ABCG2 protein expression levels by chicory were observed in jejunum and ileum. Results of the protein expression by western blotting and ABCG2 mRNA expression were consistent with the immunohistochemical staining. This showed that ABCG2 mRNA expressions in hyperuricemia rats decreased significantly to $33.85 \%$ and $42.62 \%$ in jejunum and ileum respectively, and ABCG2 protein expressions by western blotting in them decreased 
markedly to $67.08 \%$ and $74.32 \%$ (Figs. 2 and Fig. 4 ) in hyperuricemia rats. This demonstrated that excessive intake of fructose would alter the mRNA and protein expressions of $\mathrm{ABCG} 2$ in the intestine. In order to determine the underlying molecular mechanisms of uricosuric effects of chicory, the mRNA and protein expressions of ABCG2 in jejunum and ileum responsible for intestinal uric acid excretion were examined. Notably, ABCG2 mRNA expression levels of the high and middle doses of chicory were increased to $231.34 \%$ and $383.17 \%$ in jejunum, and protein expression levels of them in jejunum were markedly enhanced to $153.98 \%$ and $199.33 \%$ by western blotting, when compared with hyperuricemia rats respectively. In ileum, only the high dose of chicory increased significantly to $256.22 \%$ and $169.16 \%$ in the expressions of ABCG2 mRNA and protein respectively when compared to the model group.

This is the first report that chicory has the ability of increasing mRNA and protein expression levels of ABCG2. These findings which are combined with our previous data illustrate that chicory has multiple effects on treating hyperuricemia, including the inhibition of uric acid production, and the excretion of uric acid via the kidney and the intestine, which embodies the characteristics of multi-target and multi-channel overall treatment of traditional Chinese medicine.

\section{Conclusion}

In conclusion, chicory has the uricosuric effect in fructose-induced hyperuricemia rats, which was associated to accelerating intestinal excretion of uric acid by up-regulating the mRNA and protein expressions of $\mathrm{ABCG} 2$ in the intestine.

\section{Abbreviations \\ ABCG2: ATP-binding cassette transporter, sub-family G, member 2; BEN: Benzbromarone-treated group; $\mathrm{CHI}-\mathrm{H}$ : High-dosage chicory treated group; CHI-L: Low-dosage chicory treated group; CHI-M: Middle-dosage chicory treated group; CON: Control group; IHC: Immunohistochemistry:; MOD: Model group}

\section{Acknowledgements}

Not applicable.

\section{Funding}

This work was supported by National Natural Science Foundation of China (No.81673618), Beijing Natural Science Foundation (No.7162117),

Autonomous Subject of Graduated Student from Beijing University of Chinese Medicine (No. 2016-JYB-XS100).

\section{Availability of data and materials}

The datasets used and analyzed during the current study available from the corresponding author on reasonable request

\section{Authors' contributions}

WY carried out the animal experiments and molecular studies, host the design of the study, performed the statistical analysis and drafted the manuscript. LZJ and ZB conceived the study, and participated in its design and coordination, helped to draft the manuscript and to perform the statistical analysis. NAZ participated in optimization of HPLC protocols. BM helped to draft the manuscript and realize molecular studies. All authors read and approved the final manuscript.

\section{Competing interests}

The authors declare that they have no competing interests regarding the publication of this paper.

\section{Consent for publication}

Each author meets the criteria for authorship and assumes the

corresponding responsibility.

\section{Ethics approval}

The study protocol was approved by the Animal Care and Ethics Committee in Beijing University of Chinese Medicine.

\section{Publisher's Note}

Springer Nature remains neutral with regard to jurisdictional claims in published maps and institutional affiliations.

Received: 8 December 2016 Accepted: 29 May 2017

Published online: 13 June 2017

\section{References}

1. Keebaugh AC, Thomas JW. The evolutionary fate of the genes encoding the purine catabolic enzymes in hominoids, birds, and reptiles. Mol Biol Evol. 2010:27:1359-69.

2. Billiet L, Doaty S, Katz JD, Velasquez MT. Review of Hyperuricemia as New Marker for Metabolic Syndrome. ISRN Rheumatol. 2014; doi:10.1155/2014/852954.

3. Juraschek SP, Kovell LC, Miller ER, Gelber AC. Dose-Response Association of Uncontrolled Blood Pressure and Cardiovascular Disease Risk Factors with Hyperuricemia and Gout. PLoS One. 2013; doi:10.1371/journal.pone.0056546.

4. Mikuls TR, Farrar JT, Bilker WB, Fernandes S, Schumacher HR Jr, Saag KG. Gout epidemiology: results from the UK General Practice Research Database, 1990-1999. Ann Rheum Dis. 2005;64:267-72.

5. Wallace KL, Riedel AA, Joseph-Ridge N, Wortmann R. Increasing prevalence of gout and hyperuricemia over 10 years among older adults in a managed care population. J Rheumatol. 2004:31:1582-7.

6. Wu HW, Wang CT. Case report and analysis of allopurinol-caused ADR /ADE in 60 patients. Chinese J. New Drugs. 2013;22:2215-8.

7. Stirnimann G, Kessebohm K, Lauterburg B. Liver injury caused by drugs: an update. Swiss Med Wkly. 2010; doi:10.4414/smw.2010.13080.

8. Chaichian Y, Chohan S, Becker MA. Long-Term Management of Gout : Nonpharmacologic and Pharmacologic Therapies. Rheum Dis Clin N Am. 2014;40:357-74.

9. Li XX, Han M, Wang YY, Liu JP. Chinese herbal medicine for gout: a systematic review of randomized clinical trials. Clin Rheumatol. 2013;32:943-59.

10. Ding $X Q$, Pan $Y$, Wang $X, M a Y X$, Kong LD. Wuling san ameliorates urate under-excretion and renal dysfunction in hyperuricemic mice. Chin J Nat Med. 2013;11:214-21.

11. $\mathrm{Hu} Q \mathrm{QH}$, Jiao $R Q$, Wang $X$, Lv $Y Z$, Kong LD. Simiao pill ameliorates urate underexcretion and renal dysfunction in hyperuricemic mice. J Ethnopharmacol. 2010;128:685-92.

12. Sorensen LB. Role of the intestinal tract in the elimination of uric acid Arthritis Rheum. 1965;8:694-706.

13. Abramson RG, Levitt MF. Use of pyrazinamide to assess renal uric acid transport in the rat: a micropuncture study. Am J Phys. 1976;230:1276-83.

14. Perez-Ruiz F, Dalbeth N, Bardin T. A Review of Uric Acid, Crystal Deposition Disease, and Gout. Adv Ther. 2015;32:31-41.

15. Vitart V, Rudan I, Hayward C, Gray NK, Floyd J, Palmer CN, et al. SLC2A9 is a newly identified urate transporter influencing serum urate concentration, urate excretion and gout. Nat Genet. 2008:40:437-42.

16. Enomoto A, Kimura H, Chairoungdua A, Shigeta $Y$, Jutabha $\mathrm{P}, \mathrm{Cha} \mathrm{SH}$, et al. Molecular identification of a renal urate-anion exchanger that regulates blood urate levels. Nature. 2002:417:447-52.

17. Lopeznieto CE, You G, Bush KT, et al. Molecular cloning and characterization of NKT, a gene product related to the organic cation transporter family that is almost exclusively expressed in the kidney.[J]. J Biol Chem. 1997; 272:6471-8

18. Bakhiya A, Bahn A, Burckhardt G, Wolff N. Human organic anion transporter 3 (hOAT3) can operate as an exchanger and mediate secretory urate flux. Cell Physiol Biochem. 2003;13:249-56. 
19. Matsuo H, Yamamoto K, Nakaoka H, et al. Genome-wide association study of clinically defined gout identifies multiple risk loci and its association with clinical subtypes. Ann Rheum Dis. 2016;75:652-9.

20. Kamatani Y, Matsuda K, Okada Y, Kubo M, Hosono N, Daigo Y, et al. Genome-wide association study of hematological and biochemical traits in a Japanese population. Nat Genet. 2010:42:210-5.

21. Nigam SK. What do drug transporters really do? Nat Rev Drug Discov. 2015; 14:29-44.

22. Bhatnagar V, Richard EL, Wu W, Nievergelt CM, Lipkowitz MS, Jeff J, et al. Analysis of ABCG2 and other urate transporters in uric acid homeostasis in chronic kidney disease: potential role of remote sensing and signaling. Clin Kidney J. 2016:9:444-53.

23. Kolz M, Johnson T, Sanna S, Teumer A, Vitart V, Perola M, et al. MetaAnalysis of 28,141 Individuals Identifies Common Variants within Five New Loci That Influence Uric Acid Concentrations. PLoS Genet. 2009:5:98-111.

24. Hosomi A, Nakanishi T, Fujita T, Tamai I, et al. Extra-Renal Elimination of Uric Acid via Intestinal Efflux Transporter BCRP/ABCG2. PLoS One. 2012; doi:10.1371/journal.pone.0030456.

25. Rozpadek P, Wężowicz K, Stojakowska A, Malarz J, Surówka E, Sobczyk Ł, et al Mycorrhizal fungi modulate phytochemical production and antioxidant activity of Cichorium intybus L. (Asteraceae) under metal toxicity. Chemosphere. 2014. 112:217-24.

26. Li LY, Ling ZJ, Zhang B, Wang XJ, Zhu CS, Niu HJ, et al. Effect of Chinese Herb Chicory on Renal Organic Anion Transporter OAT3 -LIKE of Hyperuricemia Quails. Tradis.Chin. Drug Res. Clin. Pharmacol. 2015;26:284-9.

27. Wang XJ, Lin ZJ, Zhang B, Zhu CS, Niu HJ, Zhou Y, et al. Molecular docking analysis of xanthine oxidase inhibition by constituents of chicory. China J Chinese Materia Medica. 2015:40:3818-25.

28. Zhu CS, Zhang B, Lin ZJ, Wang XJ, Zhou Y, Sun XX, et al. Relationship between High-Performance Liquid Chromatography Fingerprints and Uric Acid-Lowering Activities of Cichorium intybus L. Molecules. 2015;20:9455-67.

29. Hu QH, Wang C, Li JM, Zhang DM, Kong LD. Allopurinol, rutin, and quercetin attenuate hyperuricemia and renal dysfunction in rats induced by fructose intake: renal organic ion transporter involvement. Am J Physiol Renal Physiol. 2009;297:F1080-91.

30. Ichida K, Matsuo H, Takada T, Nakayama A, Murakami K, Shimizu T, et al. Decreased extra-renal urate excretion is a common cause of hyperuricemia. Nat Commun. 2012:3:764

31. Vos MB, Kimmons JE, Gillespie C, Welsh J, Blanck HM, et al. Dietary fructose consumption among US children and adults: the Third National Health and Nutrition Examination Survey. Medscape J Med. 2008;10:160.

32. Elliott SS, Keim NL, Stern JS, Teff K, Havel PJ. Fructose, weight gain, and the insulin resistance syndrome. Am J Clin Nutr. 2002;76:911-22.

33. Basciano $\mathrm{H}$, Federico L, Adeli K. Fructose, insulin resistance, and metabolic dyslipidemia. Nutr Metab (Lond). 2005;2:1-14.

34. Fox $1 \mathrm{H}$, Kelley WN. Studies on the Mechanism of Fructose-Induced Hyperuricemia in Man. Metabolism. 1972;21:713-21.

35. Perheentupa J, Raivio K. Fructose-induced hyperuricemia. Lancet. 1967;2:528-31

36. Chen L, Lan Z, Zhou Y, Li F, Zhang X, Zhang C, et al. Astilbin attenuates hyperuricemia and ameliorates nephropathy in fructose-induced hyperuricemic rats. Planta Med. 2011;77:1769-73.

37. Hallfrisch J. Metabolic effects of dietary fructose. FASEB J. 1990:4:2652-60.

38. Smith CM, Rovamo LM, Raivio KO. Fructose-induced adenine nucleotide catabolism in isolated rat hepatocytes. Can J Biochem. 1978:55:1237-40.

39. Abdulla MH, Sattar MA, Johns EJ. The Relation between Fructose-Induced Metabolic Syndrome and Altered Renal Haemodynamic and Excretory Function in the Rat. Int J Nephrol. 2011; doi:10.4061/2011/934659.

40. Nishimura H, Kondo Y, Nagasaka T, Satoh A. Allelochemicals in Chicory and Utilization in Processed Foods. J Chem Ecol. 2000;26:2233-41.

41. Huang SN, Lin ZJ, Zhang B, Geng D, Niu HJ, Zhu CS, et al. Effect of Chicory on Uric acid and Uricopoiesis Metabolic Enzymes Activities of HYperuricemia Quail. Tradis.Chin. Drug Res. Clin. Pharmacol. 2015;26:9-13.

42. Matsuo H, Takada T, Ichida K, Nakamura T, Nakayama A, Ikebuchi Y, et al. Common Defects of ABCG2, a High-Capacity Urate Exporter, Cause Gout: A Function-Based Genetic Analysis in a Japanese Population. Sci Transl Med. 2009; doi:10.1126/scitranslmed.3000237.

43. Dehghan A, Köttgen A, Yang Q, Hwang SJ, Kao WL, Rivadeneira F, et al. Association of three genetic loci with uric acid concentration and risk of gout: a genome-wide association study. Lancet. 2008;372:1953-61.
44. Huls M, Brown CD, Windass AS, Sayer R, van den Heuvel JJ, Heemskerk S, et al. The breast cancer resistance protein transporter ABCG2 is expressed in the human kidney proximal tubule apical membrane. Kidney Int. 2008:73:220-5.

45. Maliepaard M, Scheffer GL, Faneyte IF, van Gastelen MA, Pijnenborg AC, Schinkel $\mathrm{AH}$, et al. Subcellular localization and distribution of the breast cancer resistance protein transporter in normal human tissues. Cancer Res. 2001;61:3458-64.

46. Polgar O, Robey RW, Bates SE. ABCG2: structure, function and role in drug response. Expert Opin Drug Metab Toxicol. 2007;4:1-15.

47. Yano H, Tamura Y, Kobayashi K, Tanemoto M, Uchida S. Uric acid transporter ABCG2 is increased in the intestine of the $5 / 6$ nephrectomy rat model of chronic kidney disease. Clin Exp Nephrol. 2013;18:50-5.

\section{Submit your next manuscript to BioMed Central and we will help you at every step:}

- We accept pre-submission inquiries

- Our selector tool helps you to find the most relevant journal

- We provide round the clock customer support

- Convenient online submission

- Thorough peer review

- Inclusion in PubMed and all major indexing services

- Maximum visibility for your research

Submit your manuscript at www.biomedcentral.com/submit
) Biomed Central 